МИНИСТЕРСТВО ОБРАЗОВАНИЯ И НАУКИ РОССИЙСКОЙ ФЕДЕРАЦИИ

ТОМСКИЙ ГОСУДАРСТВЕННЫЙ УНИВЕРСИТЕТ

\title{
МАТЕРИАЛЫ
}

VIII Международной молодежной научной конференции

«МАТЕМАТИЧЕСКОЕ

И ПРОГРАММНОЕ ОБЕСПЕЧЕНИЕ

ИНФОРМАЦИОННЫХ, ТЕХНИЧЕСКИХ

И ЭКОНОМИЧЕСКИХ СИСТЕМ»

Томск, 26-30 мая 2021 г.

Под общей редакиией И.С. Шмырина

Томск

Издательство Томского государственного университета 2021 


\title{
ІІ. ИСКУССТВЕННЫЙ ИНТЕЛЛЕКТ, МАШИННОЕ ОБУЧЕНИЕ, БОЛЬШИЕ ДАННЫЕ
}

DOI: $10.17223 / 978-5-907442-42-9-2021-4$

\section{КОМИТЕТНЫЕ МЕТОДЫ НЕЙРОННЫХ СЕТЕЙ В ЗАДАЧЕ РАСПОЗНАВАНИЯ РУКОПЕЧАТНЫХ СИМВОЛОВ}

\author{
Авдеев Н.Г. \\ Томский политехнический университет \\ ndb1@tpu.ru
}

Введение

Контроль знаний, умений и навыков является важным звеном учебного процесса. От его организации во многом зависит результат обучения. В процессе контроля выявляются как достоинства, так и недостатки знаний и умений учащихся, что позволяет управлять учебным процессом, совершенствуя формы и методы обучения. Массовые тестирования проводятся по регламентируемым процедурам и требуют особого подхода к организации и обработке. К такому методу контроля знаний относится и Государственная итоговая аттестация (ГИА) - форма оценки степени и уровня освоения обучающимися основных образовательных программ, соответствующим требованиям федерального государственного образовательного стандарта. Для ознакомления учеников 9 и 11 классов с заданиями ГИА, а также его процедурой проведения проводятся диагностические работы ОГЭ и ЕГЭ. Существующий формат проведения данного мероприятия ставит перед организаторами задачу анализа информации, представленной в виде изображений - произвести так называемое off-line распознавание уже написанного на бумаге текста. Задача обработки и распознавания изображений относится к разряду трудно формализуемых задач и является одной из наиболее важных на сегодняшний день.

\section{1. Выбор технологии распознавания}

Для поставленной задачи были выбраны сверточные нейронные сети. Выбор сверточных нейронных сетей обусловлен следующими факторами:

- начиная с 2012 г. сверточные сети занимают первые места на конкурсе ImageNet;

- сверточные нейронные сети устойчивы к аффинным преобразованиям;

- сверточные нейронные сети учитывают топологию изображений [1].

\section{2. Подготовка обучающей выборки}

Выборка подготовлена из бланков ЕГЭ прошлых лет. Исходная выборка состоит из 47850 монохромных изображений размером 32х32 (по 1450 изображений для каждого класса буквы). Примеры изображений продемонстрированы на рис. 1.

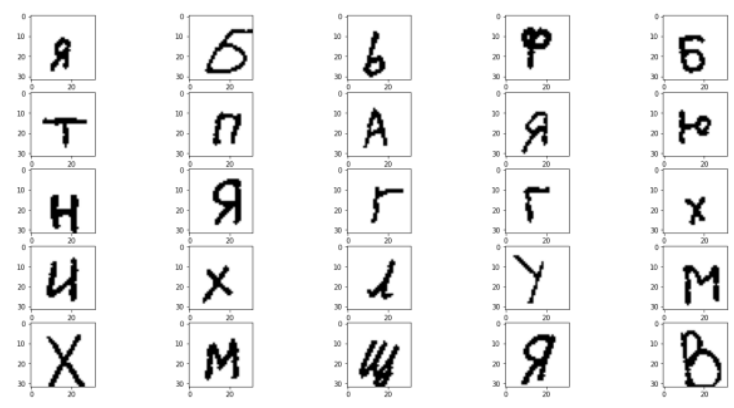

Рис. 1. Примеры изображений букв 
Выборка была разделена на тренировочную, валидационную и тестовую в соотношении $70 \%, 20 \%$ и $10 \%$ соответственно [2].

\section{3. Вычислительные эксперименты}

Для экспериментов была выбрана модель сверточной нейронной сети LeNet5 [3]. Количество нейронов на выходном слое было увеличено до 33 (по количеству классов). Данная нейронная сеть хорошо себя зарекомендовала в задачах распознавания рукописных символов. Архитектура LeNet5 для распознавания рукописных цифр продемонстрирована на рис. 2.

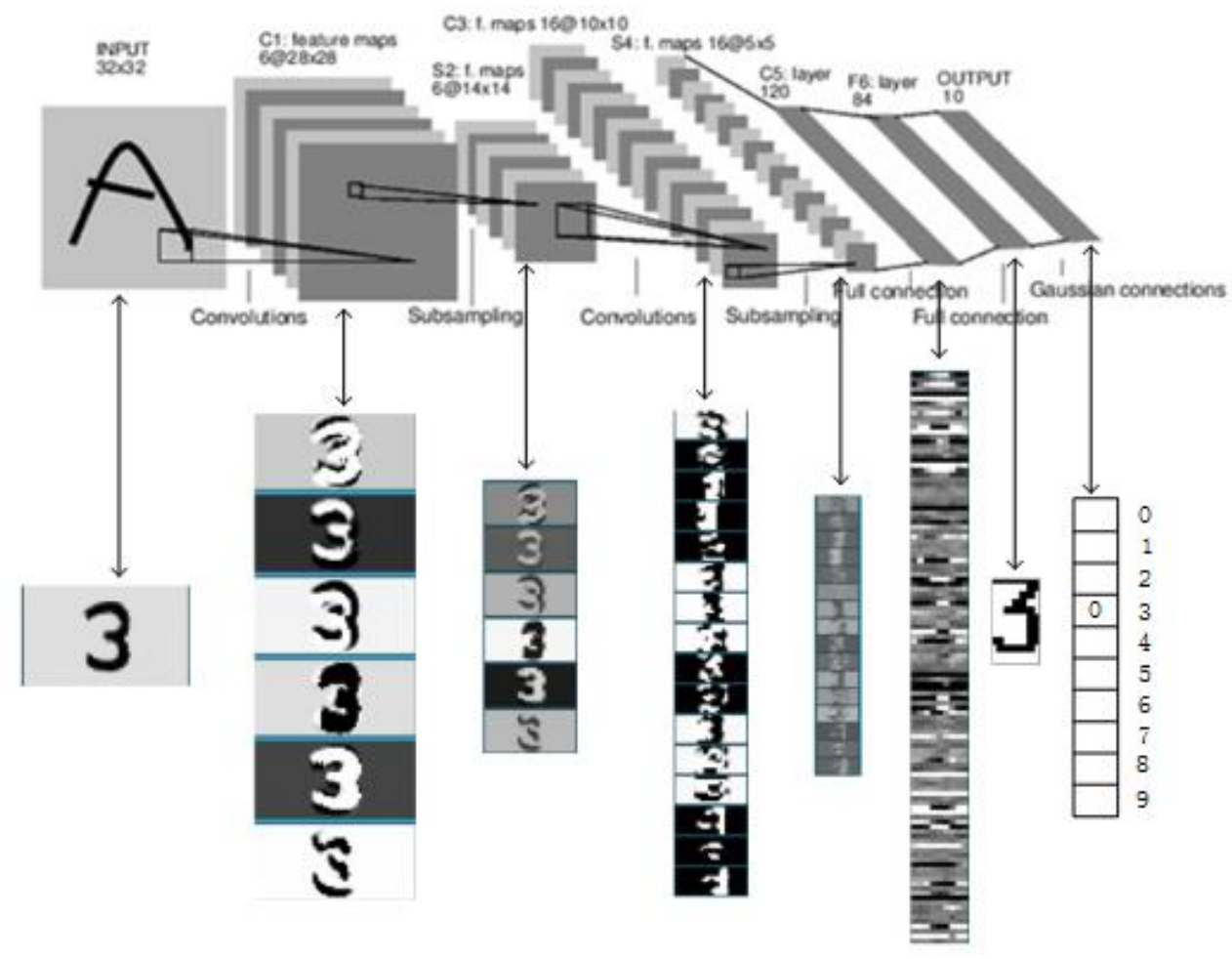

Рис. 2. Архиектура сверточной сети LeNet5

Нейронная сеть была обучена на тренировочной выборке. Точность обученной нейронной сети составляет $94.5 \%$.

\section{4. Метод бэггинга}

Для повышения качества распознавания символов предлагается использовать композицию нейронных сетей с архитектурой LeNet5 . Одним из способов получения композиции классификаторов является использование бэггинга (bootstrap aggregation). Данный метод заключается в независимом обучении нескольких моделей на подвыборках, полученных из исходных с помощью статистического метода под названием бутстрэп. Этот статистический метод заключается в генерации выборок размера $B$ (так называемых бутстрэп-выборок) из исходного набора данных размера $N$ путем случайного выбора элементов с повторениями в каждом из наблюдений $B$. Метод бэггинга позволяет получить ансамблевую модель с меньшим разбросом, чем ее отдельные компоненты [4].

Результирующее предсказание классификатора может быть получено одним из следующих способов: 
- консенсус: если все элементарные классификаторы присвоили объекту одну и ту же метку, то относим объект к выбранному классу;

- простое большинство: объекту метка того класса, который определило для него большинство элементарных классификаторов;

- взвешивание классификаторов: при голосовании голос классификатора умножается на его вес, отражающий его качество [5].

На практике консенсус достижим очень редко и это приводит к уменьшению точности распознавания, поэтому было решено реализовать методы простого большинства и взвешивания классификаторов. В методе взвешивания классификаторов использовался подход, заключающийся в нахождении результирующего значения классификации на основании точности распознавания сети каждого из классов. еще одним настраиваемым параметром является размер бутстрэп-выборок. Будем выбирать размер бутстрэпвыборки равным от $10 \%$ до 90\% от тестовой выборки с шагом, равным $10 \%$. Для каждой из бутстрэп-выборок будем находить точность для ансамблей, состоящих из разного количества нейронных сетей (от 1 до 10). На рис. 3 продемонстрированы результаты вычислительного эксперимента.
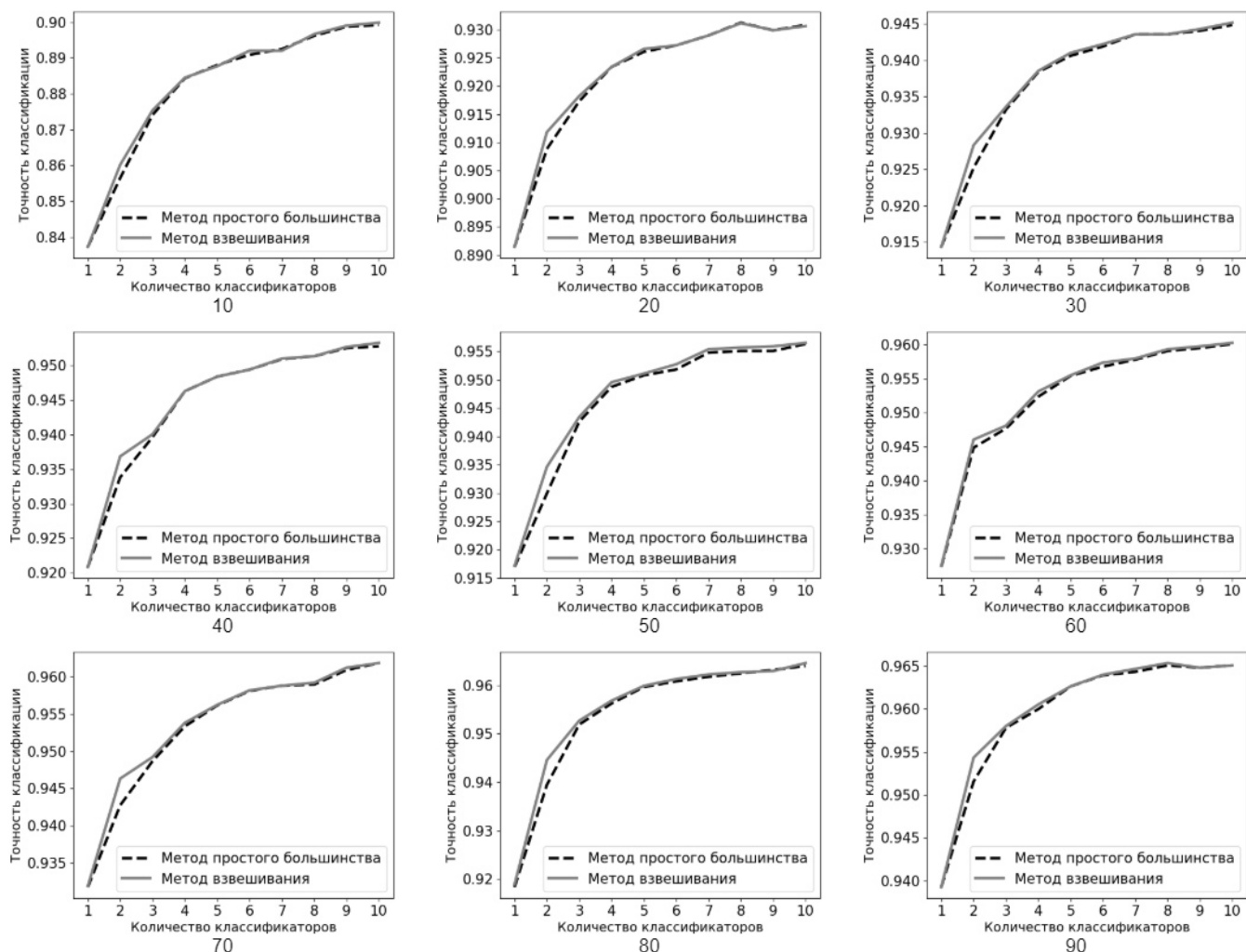

Рис.3. Результаты эксперимента для бэггинга

Каждый график на рис. 3 соответствует вычислительному эксперименту с бутстрэп-выборкой определенного размера (подпись под графиком). По оси абсцисс отложены значения размера ансамбля нейронных сетей, по оси ординат - значения точности распознавания для данного ансамбля. Результирующее значение точности вычислялось как среднее по 100 запускам. Серой линии на графике соответствует метод взвешивания классификаторов, черной пунктирной - метод простого большинства. 
Наибольшая точность классификации, равная 96.5\%, была достигнута при размере обучающей выборки для классификаторов, равном 90\% от количества примеров в обучающей выборке, и количестве классификаторов, равном 8.

\section{5. Метод бустинга}

Другим способом получения композиции классификаторов является бустинг. Бустинг заключается в построении композиции алгоритмов, когда каждый последующий алгоритм стремится компенсировать недостатки предыдущих алгоритмов. Одними из самых известных алгоритмов бустинга на сегодняшний день являются градиентный бустинг, который используется в основном для решающих деревьях (и его различные модификации), и AdaBoost. Принцип работы алгоритма AdaBoost заключается в том, что обучающим примерам присваиваются веса, в зависимости от того, корректно или некорректно они классифицировались. Существует два алгоритма для вычисления веса обучающего примера: SAMME и SAMME.R. SAMME.R использует оценки вероятности принадлежности к классам, SAMME использует только факт верной/неверной классификации. Большие веса получают неверно классифицированные примеры. Примеры с большими весами имеют больший шанс попадания в обучающую выборку для следующего классификатора [6].

От количества эпох обучения зависит то, насколько хорошо сеть «запомнила» обучающие примеры, и, следовательно, сколько примеров попадет в обучающую выборку для следующего классификатора. Слишком большое количество эпох обучения может привести к переобучению сети, слишком маленькое - к недообучению. Будем выбирать количество эпох от 10 до 40 с шагом 10. Еще одним оптимизируемым параметром является количество классификаторов. На рис. 4 продемонстрированы результаты вычислительного эксперимента.

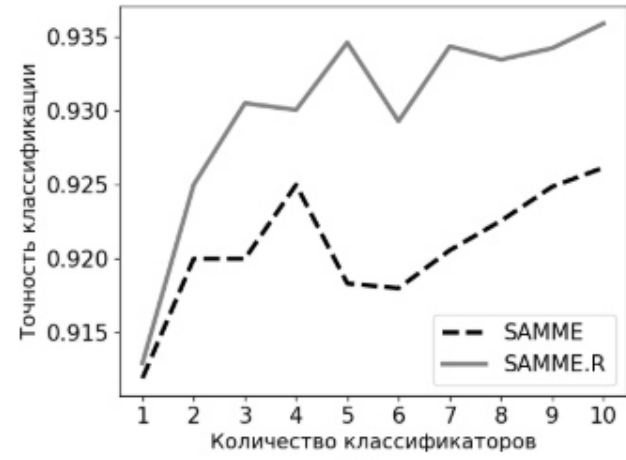

10

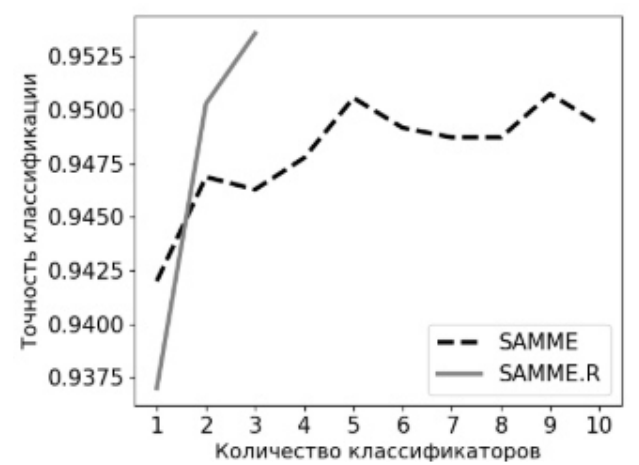

30

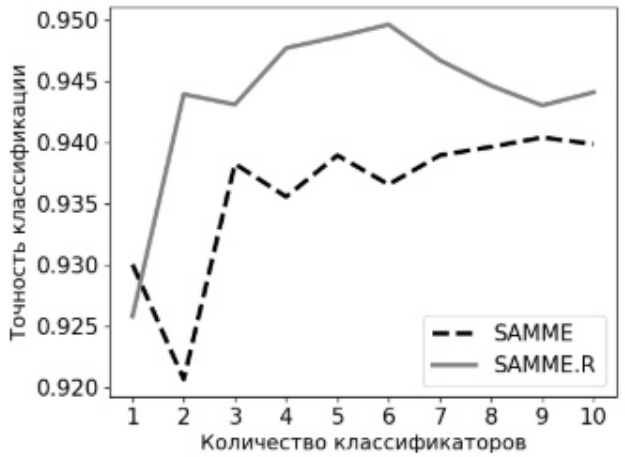

20

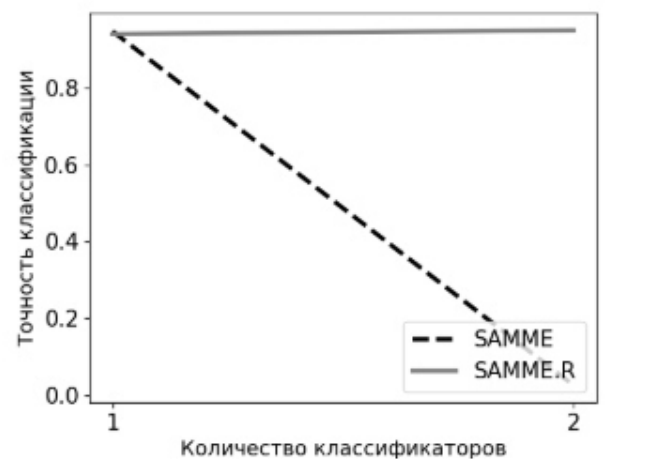

40

Рис. 4. Результаты эксперимента для бустинга 
Каждому графику на рис. 4 соответствует вычислительный эксперимент для определенного количества эпох (подпись под графиком). По оси абсцисс отложены значения размера ансамбля нейронных сетей, по оси ординат - значения точности распознавания для данного ансамбля. Результирующее значение точности вычислялось как среднее по 100 запускам. Серой линии на графике соответствует алгоритм SAMME.R, черной пунктирной - алгоритм SAMME. Алгоритм прекращает работу, когда ошибка на тренировочной выборке становится равной 0 , что продемонстрировано на графиках для количества эпох, равных 30 и 40.

Как видно из результатов эксперимента, алгоритм SAMME.R демонстрирует большую точность, чем алгоритм SAMME. Наибольшая точность (95.4\%) была достигнута для трех классификаторов в комитете, количества эпох, равных 30 , и алгоритма SAMME.R.

\section{Заключение}

В результате работы была реализованы композиция классификаторов сверточных нейронных сетей архитектуры LeNet5 на основании методов бэггинга и бустинга. Работа композиции оценивалась на выборке рукописных символов, полученных с бланков ЕГЭ. В результате работы были сделаны следующие выводы:

1. Метод бустинга позволил достичь максимальной точности классификации $95.4 \%$ на основе композиции из 3-х классификаторов сверточных нейронных сетей архитектуры LeNet5.

2. Алгоритм SAMME.R демонстрирует большую точность, чем алгоритм SAMME.

3. Метод бэггинга позволил достичь максимальной точности классификации $96.5 \%$ на основе композиции из 8-ми классификаторов сверточных нейронный сетей архитектуры LeNet5.

4. Предложенная композиция увеличила точность распознавания на $2 \%$ по сравнению с одним классификатором LeNet5.

5. Вместе с размером бутстрэп-выборки растет значение точности распознавания ансамблей.

6. Метод взвешенных классификаторов демонстрирует незначительно большую точность для ансамблей, состоящих из двух классификаторов.

\section{ЛИТЕРАТУРА}

1. Сверточная нейронная сеть, часть 1: структура, топология, функции активации и обучающее множество // [Электронный pecypc]. URL: https://habr.com/ru/post/348000/ (Дата обращения: 14.06.2021).

2. The Train, Validation, Test Split and Why You Need It. // [Электронный pecypc]. URL: https://blog.roboflow.com/train-test-split/ (Дата обращения: 14.06.2021).

3. Understanding and Implementing LeNet-5 CNN Architecture (Deep Learning) // [Электронный ресурс]. URL https://towardsdatascience.com/understanding-and-implementing-lenet-5-cnn-architecture-deep-learning-a2d531ebc342 (Дата обращения: 14.06.2021).

4. Ансамблевые методы: бэггинг, бустинг и стекинг // [Электронный pecypc]. URL: https://neurohive.io/ru/osnovy-data-science/ansamblevye-metody-begging-busting-i-steking/ (Дата обращения: 14.06.2021).

5. Бэггинг // [Электронный pecypc]. URL: http://www.machinelearning.ru/wiki/index.php?title=Бэггинг (Дата обращения: 14.06.2021).

6. Multi-class AdaBoost // [Электронный pecypc]. URL: https://web.stanford.edu/ hastie/Papers/samme.pdf (Дата обращения: 14.06.2021). 